\title{
Cellular entry of nanoparticles via serum sensitive clathrin-mediated endocytosis, and plasma membrane permeabilization
}

This article was published in the following Dove Press journal:

International Journal of Nanomedicine

2I April 2012

Number of times this article has been viewed

\author{
Philip J Smith' \\ Maude Giroud ${ }^{2}$ \\ Helen L Wiggins ${ }^{2}$ \\ Florence Gower ${ }^{2}$ \\ Jennifer A Thorley² \\ Bjorn Stolpe ${ }^{3}$ \\ Julie Mazzolini² \\ Rosemary J Dyson ${ }^{4}$ \\ Joshua Z Rappoport ${ }^{2}$
}

'Physical Sciences of Imaging for the Biomedical Sciences (PSIBS) Doctoral Training Center, School of Chemistry, ${ }^{2} \mathrm{~S}$ chool of Biosciences, ${ }^{3} \mathrm{~S}$ chool of Geography, Earth, and Environmental Sciences, ${ }^{4}$ School of Mathematics, University of Birmingham, Edgbaston, Birmingham, United Kingdom
Correspondence: Joshua Z Rappoport School of Biosciences, University of Birmingham, Edgbaston, Birmingham BI5 2TT, United Kingdom Tel +44 I2I 4149019

Email j.rappoport@bham.ac.uk

\begin{abstract}
Increasing production and application of nanomaterials raises significant questions regarding the potential for cellular entry and toxicity of nanoparticles. It was observed that the presence of serum reduces the cellular association of $20 \mathrm{~nm}$ carboxylate-modified fluorescent polystyrene beads up to 20 -fold, relative to cells incubated in serum-free media. Analysis by confocal microscopy demonstrated that the presence of serum greatly reduces the cell surface association of nanoparticles, as well as the potential for internalization. However, both in the presence and absence of serum, nanoparticle entry depends upon clathrin-mediated endocytosis. Finally, experiments performed with cells cooled to $4{ }^{\circ} \mathrm{C}$ suggest that a proportion of the accumulation of nanoparticles in cells was likely due to direct permeabilization of the plasma membrane.
\end{abstract}

Keywords: nanoparticles, polystyrene beads, serum, endocytosis, dynamin, clathrin, permeabilization

\section{Introduction}

Nanoparticles are defined as structures with at least one dimension smaller than $100 \mathrm{~nm} .{ }^{1}$ Although humans have always come into contact with naturally occurring nanoparticles, the ever increasing production and application of manufactured nanoparticles in industry, research, and medicine has led to greater potential for incidental environmental contact, as well as deliberate exposure through products and therapeutics. ${ }^{2,3}$ However, the potential for cellular entry and toxicity of manufactured nanoparticles has only recently begun to be investigated. The extent of potential cellular effects following nanoparticle exposure can depend upon target cell type, environmental conditions, nanoparticle composition, size, shape, surface chemistry, and dosage. ${ }^{4}$

Given the potential for heterogeneity, when comparing different nanoparticles, cells, and experimental conditions, each particular system of interest must be empirically analyzed. Therefore, model nanoparticles with well-defined characteristics have been serving as a valuable tool in understanding the potential for intracellular exposure and cellular toxicity. ${ }^{5-9}$ Polystyrene nanoparticles can be generated (eg, FluoSpheres ${ }^{\circledR}$ from Invitrogen Life Technologies, Carlsbad, CA) within accurate size ranges, to a nearly uniform shape, and with a variety of surface coatings and fluorescent labels useful for tracking in biological systems. Already, numerous studies have been published analyzing the entry of polystyrene nanoparticles into different types of cells; however, these reports have not been entirely consistent in the observations reported.$^{5-9}$ In particular, size and surface characteristics have emerged as potentially important characteristics regulating the potential for cellular entry and the specific mechanisms involved. ${ }^{4-10}$ 
One further factor which could affect the potential for cellular entry of nanoparticles is the generation of a "protein corona." It has been determined that carboxylate modification of polystyrene nanoparticles can promote interactions with proteins, ${ }^{11}$ and reduced cell surface binding was observed for larger polystyrene particles (eg, $\sim 200 \mathrm{~nm}$ ) incubated in the presence of serum. ${ }^{12}$ Therefore, studies were performed to assess potential serum-dependent effects on nanoparticle interactions with cells, and potential mechanisms for nanoparticle internalization. Taken together, the results demonstrate a striking serum dependence for plasma membrane binding and subsequent nanoparticle entry into cells via clathrin-mediated endocytosis. Finally, these studies suggest that plasma membrane permeabilization is another potential mode of nanoparticle entry into cells. This is significant when considering other nanomaterials that are used for humans as this effect was seen both in the presence and absence of serum, suggesting this effect may be size dependent only.

\section{Material and methods}

\section{Cell culture}

HeLa and MDCK cell lines (Health Protection Agency Culture Collections, Salisbury, United Kingdom) were grown at $37^{\circ} \mathrm{C}$ in the presence of $5 \%$ carbon dioxide with Gibco $^{\circledR}$ Dulbecco's modified Eagle medium (DMEM) (Invitrogen) supplemented with 10\% fetal calf serum (Invitrogen), and $1 \%$ penicillin/streptomycin (Invitrogen). Cells were detached with trypsin (Invitrogen) following Gibco phosphate buffered saline (PBS) wash (Invitrogen). Detached cells were transferred at appropriate dilutions into new flasks or plated onto $24 \mathrm{~mm}$ glass coverslips in six-well plates for fixation. They were incubated in a $\mathrm{CO}_{2}$ ncubator (MCO-17AIC; SANYO, Osaka, Japan) at $37^{\circ} \mathrm{C}$ in the presence of $5 \%$ carbon dioxide for $\sim 24$ hours prior to transfection (see below) or incubation in nanoparticles (see below).

\section{Nanoparticle uptake experiments: plate reader}

Cells were grown in $\mu$ Clear ${ }^{\circledR}$ black 96 -well glass bottom plates (Greiner Bio-One GmbH, Frickenhausen, Germany). Yellow/green and red $20 \mathrm{~nm}$ carboxylate-modified polystyrene FluoSpheres (Invitrogen) (stock $0.02 \mathrm{~g} / \mathrm{mL} \equiv 4.54 \times 10^{15}$ nanoparticles $/ \mathrm{mL}$ ) were at a $1 \%$ dilution in either serum-containing or serum-free media and added to cells for $0,5,15,30,60$, and 120 minutes. After the allotted time, cells were rinsed twice with $100 \mu \mathrm{L}$ PBS and incubated for 5 minutes in $100 \mu \mathrm{L}$ of $4 \%$ paraformaldehyde (Electron Microscopy Sciences, Hatfield,
PA) in PBS. They were then rinsed twice more with $100 \mu \mathrm{L}$ PBS. The fluorescence was observed with PBS solution inside the wells with a FLUOStar Omega fluorescence plate reader (BMG LABTECH GmbH, Ortenberg, Germany).

\section{Determination of size and zeta potential by dynamic and electrophoretic light scattering}

Size (hydrodynamic diameter) and zeta potential measurements of nanoparticle dispersion were done using a Zetasizer ${ }^{\circledR}$ Nano ZS ZEN3600 (Malvern Instruments Ltd, Malvern, United Kingdom) to investigate possible aggregation of nanoparticles. Nanoparticles were at $0.5 \%$ dilution from stock in each experimental variation and sonicated in ultrasonic bath (XUB18; Grant Instruments, Shepreth, UK) for 20 minutes before measurement.

\section{Nanoparticle cytotoxicity experiments}

Cells were grown on MatTek glass bottom plates (MatTek Corporation, Ashland, MA) for 24 hours in $2 \mathrm{~mL}$ serumcontaining media. The media was exchanged for one of the following: serum-containing media; serum-free media; serum-containing media with $1 \%$ nanoparticles from stock solution; or serum free media with $1 \%$ nanoparticles from stock solution for 15 minutes at $37^{\circ} \mathrm{C}$. These were then replaced with serum-containing media and returned to the incubator for a further 24 or 72 hours. For counting, the cells were rinsed twice with $2 \mathrm{~mL}$ PBS and incubated in $1 \mathrm{~mL}$ trypsin for 4 minutes. Cells were put into a Hawksley counting chamber (AS1000 Improved Neubauer Double Cell Standard; Hawksley, Sussex, United Kingdom) and counted.

\section{Nanoparticle uptake experiments: microscopy}

Cells were attached onto glass coverslips and incubated overnight. For the $4^{\circ} \mathrm{C}$ experiment, cells were put on ice for 5 minutes in either serum-containing or serum-free media. Chilled green nanoparticles diluted to $1 \%$ in either serumcontaining or serum-free media were introduced to the cells for 15 minutes. For fixation, cells were rinsed in $2 \mathrm{~mL}$ chilled PBS twice and fixed with $1 \mathrm{~mL} 4 \%$ paraformaldehyde in PBS for 5 minutes. At this time the chilled cells were brought back to room temperature. They were then rinsed twice more with $2 \mathrm{~mL}$ PBS and then placed cells down on a coverslip with a small drop of Vectashield ${ }^{\circledR}$ containing 4',6-diamidino-2-phenylindole (Vector Laboratories Ltd, Peterborough, United Kingdom). They were then held in 
place using a small coat of nail varnish around the edge and stored in a refrigerator.

\section{Dynasore inhibition of nanoparticle uptake}

The following day after attachment to glass coverslips, cells were rinsed twice with warm DMEM and incubated with $2 \mathrm{~mL}$ per well of DMEM and a $0.1 \%$ dilution of dynasore (Sigma-Aldrich Corporation, St, Louis, MO) from $80 \mathrm{mM}$ stock or $0.1 \%$ dilution of dimethyl sulfoxide (Sigma-Aldrich) from $5 \mu \mathrm{g} / \mathrm{mL}$ stock. After 15 minutes the media was removed and replaced with: $1 \mathrm{~mL}$ of either DMEM or DMEM with $10 \%$ fetal calf serum; $0.1 \%$ dilution of dynasore or dimethyl sulfoxide; and $1 \%$ red FluoSpheres, which were then incubated for 15 minutes. Cells were then rinsed with PBS, fixed, and imaged as below.

\section{Nanoparticle uptake studies in dominant negative transfected cells}

Cells were transfected with $4 \mu \mathrm{g}$ of either green fluorescent protein (GFP; Clontech Laboratories Inc, Mountain View, CA), EH29-GFP (provided by Alexandre Benmerah, Institut Cochin, Paris, France), or Caveolin1(Y14F)-GFP (provided by Mark McNiven, Mayo Clinic, Rochester, MN) using Lipofectamine ${ }^{\mathrm{TM}} 2000$ (Invitrogen) according to the manufacturer's protocol. Nanoparticle uptake assays were conducted the following day with two $2 \mathrm{~mL}$ PBS rinses followed by a 15-minute incubation with DMEM, which was then removed. Cells were incubated with a $1 \%$ dilution (from stock $0.2 \mathrm{mg} / \mathrm{mL}$ ) of $20 \mathrm{~nm}$ carboxylate-modified polystyrene red FluoSpheres in either $1 \mathrm{~mL}$ of DMEM or DMEM with $10 \%$ fetal calf serum media for 15 minutes and washed twice in $2 \mathrm{~mL}$ PBS. Coverslips were fixed as above.

\section{Transferrin uptake experiments}

Cells were attached onto glass coverslips and incubated overnight. For the $4^{\circ} \mathrm{C}$ experiment, cells were put on ice for 10 minutes in either serum-containing or serum-free media. Chilled transferrin (Alexa Fluor 488-labeled; Invitrogen) diluted to $0.2 \%$ in serum-free media were introduced to the cells for 15 minutes. For fixation, cells were rinsed in $2 \mathrm{~mL}$ chilled PBS twice and fixed with $2 \mathrm{~mL} \mathrm{4 \%}$ paraformaldehyde for 5 minutes. Chilled cells were then brought back to room temperature. They were then rinsed twice more with $2 \mathrm{~mL}$ PBS and then placed cells down on a coverslip with a small drop of Vectashield containing 4',6-diamidino-2- phenylindole. They were then held in place using a small coat of nail varnish around the edge and stored in a refrigerator.

\section{Sytox ${ }^{\circledR}$ Green (Invitrogen)}

The Sytox Green for the nanoparticle and control conditions was in serum-free or serum-containing media during incubation at a concentration of $0.02 \%$. Alternatively, the fixed cells were incubated for 5 minutes in $0.1 \%$ Triton X100 solution (Sigma-Aldrich Corporation, St Louis, MO) and then incubated for 5 minutes in a $0.02 \%$ Sytox Green solution (Invitrogen, Grand Island, NY) before mounting.

\section{Imaging and image analysis}

Images for the comparison of nanoparticle effects at $4^{\circ} \mathrm{C}$ and $37^{\circ} \mathrm{C}$ were taken on an A1R inverted confocal microscope (Nikon Corporation, Tokyo, Japan) using a $60 \times$ oil immersed objective. All other images were taken using an LSM 710 confocal microscope (Carl Zeiss Microscopy GmbH, Oberkochen, Germany) using $488 \mathrm{~nm}$ and $543 \mathrm{~nm}$ lasers with a $40 \times$ or $60 \times$ oil objective lens. All analyses were conducted using NIS-Elements 3.2 imaging software (Nikon). A cell that wasn't in contact with any others was selected, and an image from the center of the confocal Z-stack was chosen. Total intensity of fluorescence per nanoparticle spot (defined as the visible intracellular accumulations of nanoparticles) was calculated by drawing around each spot and taking intensity values for it. The intracellular background fluorescence was subtracted (calculated from an area adjacent to the spot, of equal size). The number of nanoparticle spots per cell area was calculated by counting the number of visible nanoparticle spots in the slice chosen, and dividing it by the area of the cell in that slice. Any spots that lay on the cell membrane were not counted. Each experiment was performed three times, and from each experiment ten cells were analyzed.

\section{Results}

\section{Serum effects}

Previous studies have demonstrated a role for endocytosis pathways in the cellular entry of polystyrene nanoparticles. ${ }^{5-9}$ Cells are conventionally cultured in the presence of $10 \%$ fetal calf serum; however, most assays for receptor-mediated endocytosis are performed in serum-free conditions. ${ }^{13-15}$ Furthermore, it has been previously suggested that the formation of a protein corona around nanoparticles might reduce membrane adsorption, ${ }^{10,16}$ and that interaction with the membrane may be important in permitting cellular entry. ${ }^{12,17}$ Therefore, the potential for serum-dependent cellular 
A

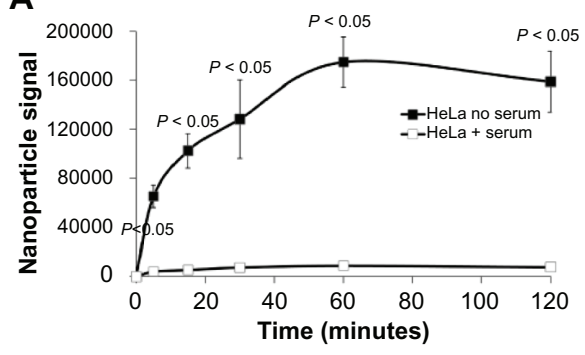

C

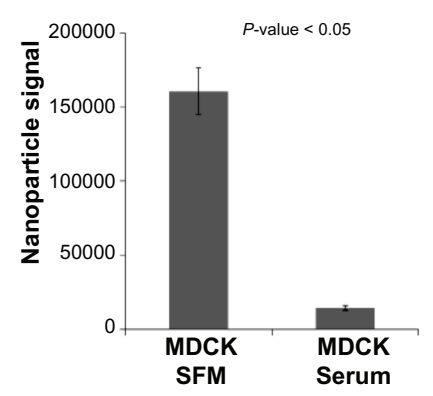

D

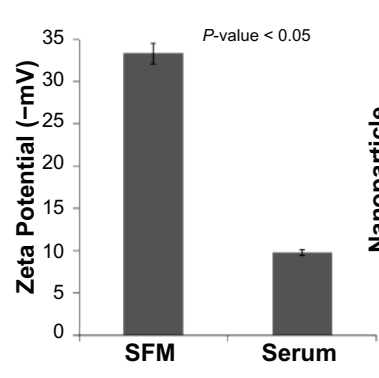

B

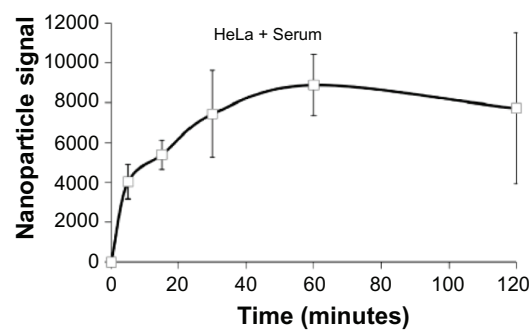

E

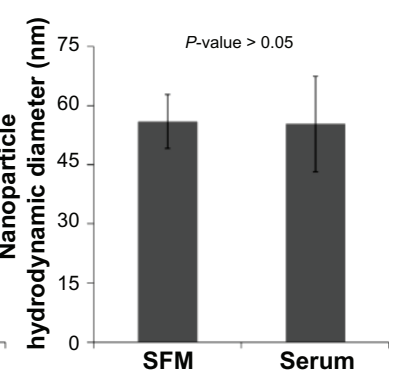

Figure I The addition of serum to cells incubated with nanoparticles results in up to a 20-fold decrease in associated cellular fluorescence and a three-fold decrease in zeta potential, without affecting nanoparticle accumulation size compared to those incubated without serum. (A and B) Nanoparticle fluorescence signal associated with HeLa cells incubated with nanoparticles in either serum-free or serum-containing media over the course of 120 minutes. (C) Nanoparticle fluorescence signal associated with MDCK cells incubated with nanoparticles in either serum-free or serum-containing media after 60 minutes. (D) Zeta potential associated with nanoparticles in either serumfree or serum-containing media. (E) Average nanoparticle hydrodynamic diameter (Z-average size) in either serum-free or serum-containing media.

Abbreviation: SFM, serum-free media.

interactions of a well-characterized type of nanoparticle ( $20 \mathrm{~nm}$ carboxylate-modified polystyrene beads), which are commercially available with a variety of fluorescent tags, was evaluated.

HeLa human carcinoma cells were incubated with nanoparticles in the presence or absence of serum. As depicted in Figure 1, analysis of cellular-associated nanoparticle signal in a fluorescence plate reader demonstrated striking serum dependence. In both the presence and absence of serum, a time-dependent cellular nanoparticle signal was observed. However, at each time point evaluated the addition of serum resulted in an up to 20-fold decrease in cellular-associated nanoparticle signal. Similar effects were observed with MDCK canine epithelial cells (Figure 1C), suggesting that this effect is neither cell type nor species dependent.

One possible explanation for the observed serum dependence in cellular association of nanoparticles would be that the serum proteins are causing the nanoparticles to aggregate, which might prevent cellular binding and/or entry. However, previously published dynamic light scattering data demonstrate that serum does not cause aggregation of carboxylatemodified polystyrene nanoparticles. ${ }^{18}$ Furthermore, this was verified in the dynamic light scattering measurements of the current study (Figure 1E). Another possible explanation for the observed effects could be due to serum directly reducing the fluorescence of the nanoparticles. However, time-lapse confocal microscopy demonstrates that the addition of serum to nanoparticles incubated in the absence of cells did not affect nanoparticle fluorescence (Figure 2). The particles depicted within a single field were individually quantified over time and no overall decrease in fluorescence was observed. Thus, it can be concluded that serum is directly reducing the potential of nanoparticles to interact with cells, rather than the fluorescence of the nanoparticles. One possible explanation for this can be the serum effects on the zeta potential of the nanoparticles (Figure 1D). The reduced negative charge may be a major contributing factor in the ability of nanoparticles to bind to the cell membrane.

One limitation of assays performed in a fluorescence plate reader is that it cannot readily distinguish between fluorescence associated with the cell surface and intracellular signal. Therefore, to further analyze the effects of serum upon the interactions of nanoparticles with cells, confocal microscopy studies were performed. As depicted in Figure 3, when imaged with identical laser power and gain settings, both the cell surface binding and the intracellular accumulation of nanoparticles was clearly higher in cells incubated in serum-free media when compared to cells exposed to nanoparticles in the presence 

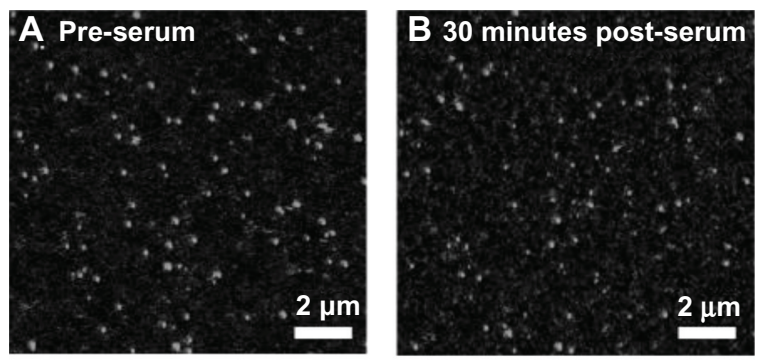

C

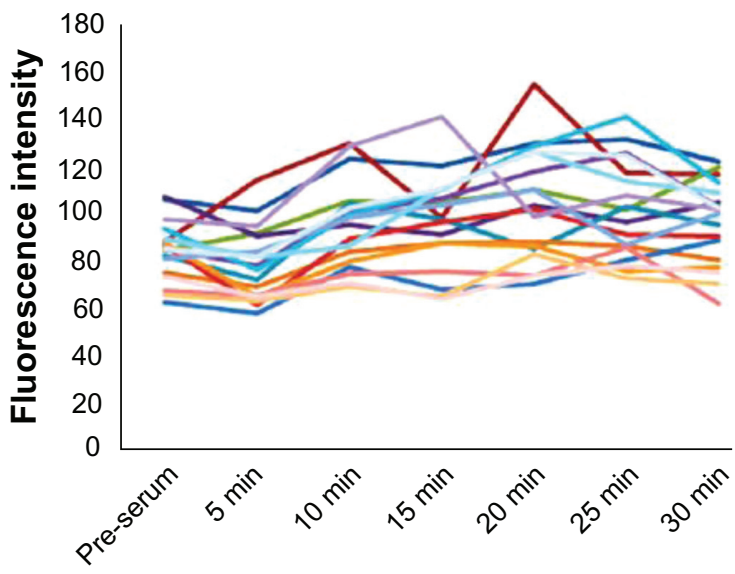

Figure 2 The addition of serum to nanoparticles in the absence of cells does not affect nanoparticle fluorescence. Representative images of nanoparticles in (A) serum-free media and (B) after 30 minutes incubation with serum. (C) Total fluorescence intensity of nanoparticle spots in a single frame over 30 minutes.

of $10 \%$ serum. Quantification of the brightness of numerous intracellular nanoparticle accumulations reveals significantly higher fluorescence in the serum-free group compared to the serum group (Figure 3C). The higher fluorescence seen on the cell membrane can also be seen (Figure 3E). It should be noted that the blue 4',6-diamidino-2-phenylindole channel gain and exposure times were adjusted to facilitate the identification of cells, hence the discrepancy in appearance.

Although the precise intracellular compartment(s) within which the nanoparticles are present is not currently known, the apparent size and brightness is potentially consistent with endosomal localization. Furthermore, when compared with the nanoparticles imaged in vitro (Figure 2), it is clear that the intracellular nanoparticle accumulations are much larger than the potentially monodisperse population adhered to the coverslip. Interestingly, it can also be seen from these images that accumulation of nanoparticles in the nucleus was not observed. Finally, the number of intracellular nanoparticle accumulations per unit area was no different between the two conditions (Figure 3D), suggesting that although the total amount of nanoparticles able to enter cells is lower in the presence of serum, in both the presence and absence of serum the nanoparticles might be entering cells via the same pathway(s).
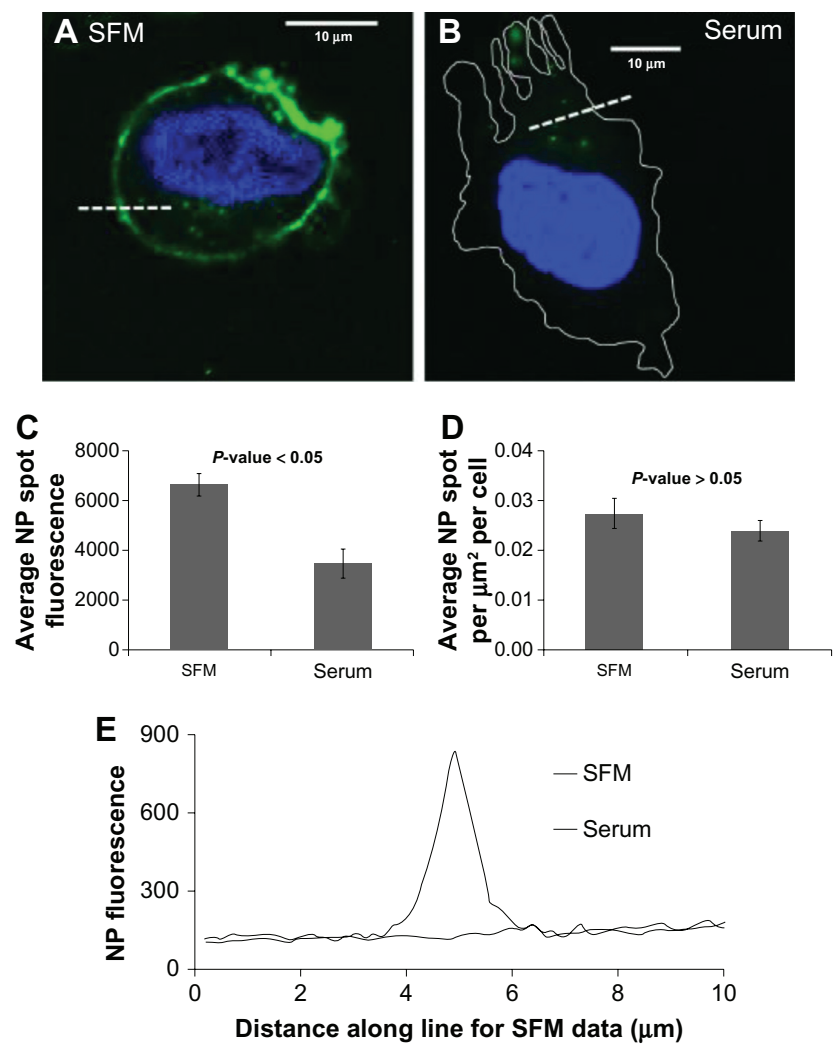

Figure 3 Both cell surface binding and intracellular accumulation of nanoparticles is higher in cells incubated in serum-free media. Representative images of a HeLa cell incubated with nanoparticles in (A) serum-free media and (B) serum-containing media. (C) Average nanoparticle spot fluorescence for cells incubated in serum-free or serum-containing media. There is a significant decrease in fluorescence for cells incubated in serum. (D) Average spot density for cells incubated in serum-free or serum-containing media. There is no difference between the two. (E) Line scan profiles of the cell membrane demonstrate greatly increased cell surface nanoparticle binding in serum-free media relative to serum-containing media.

Abbreviations: NP, nanoparticle; SFM, serum-free media.

A cytotoxicity assay was performed to evaluate whether increased cell surface binding of nanoparticles has more of a detrimental effect on cells. As seen in Figure 4, cells exposed to these polystyrene nanoparticles display a moderate degree of cytotoxicity. An approximately $30 \%$ decrease in cell numbers was observed relative to control at both 24 and 72 hours following nanoparticle treatment, both when serum was present and absent at the time of exposure. However, due to internal variance and the modest effect observed, this was only statistically significant in the 24-hour serum-free and 72-hour serum-containing conditions.

\section{Endocytosis studies}

Different pathways have been implicated in the cellular entry of nanoparticles depending upon the particular cell line used and/or the physiochemical characteristics of the specific particles being tested (eg, size, composition, or sur- 
A

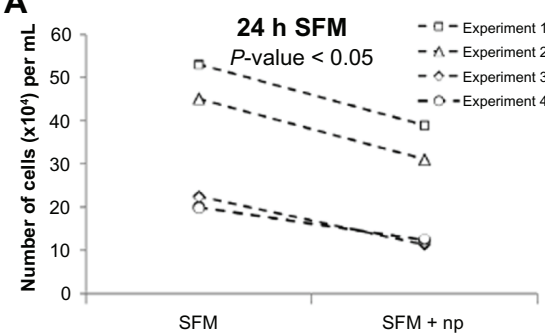

C

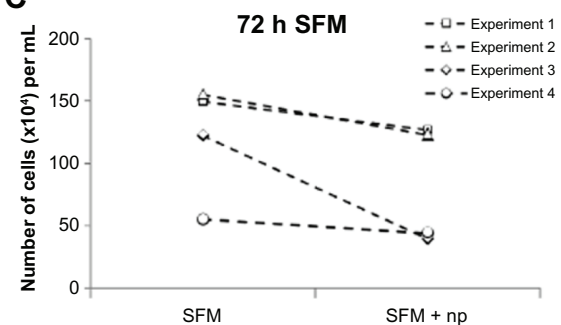

B

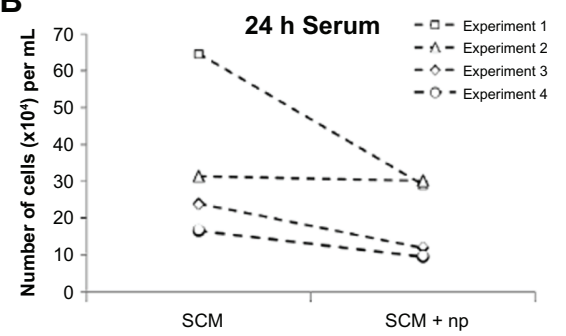

D

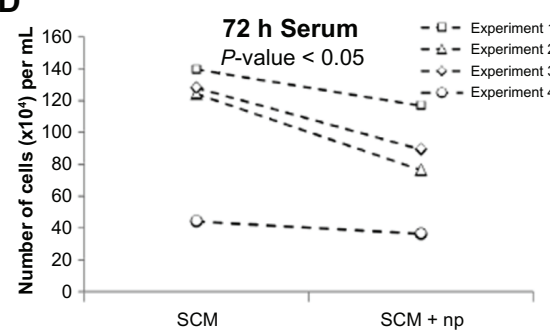

Figure 4 Nanoparticles have a minor cytotoxic effect on cells in both serum-containing and serum-free conditions. Graphs displaying the effects of incubating cells in ( $\mathbf{A}$ and $\mathbf{C})$ serum-free media with and without nanoparticles and (B and $\mathbf{D})$ serum-containing media with and without nanoparticles. (A and $\mathbf{B})$ Twenty-four hours and (C and D) 72 hours after a I5-minute incubation.

Abbreviations: h, hours; np, nanoparticle; SCM, serum-containing media; SFM, serum-free media.

face characteristics). ${ }^{4-8}$ Therefore, the aim was to empirically determine which endocytosis pathway(s) might be involved in entry of $20 \mathrm{~nm}$ carboxylate-modified polystyrene beads in HeLa cells. Dynamin is a large guanosine triphosphatase which regulates several endocytosis pathways and is potently and specifically inhibited by the small molecule dynasore. ${ }^{19,20}$ As depicted in Figure 5, in the absence of serum, dynasore treatment results in a clear inhibition of nanoparticle entry.
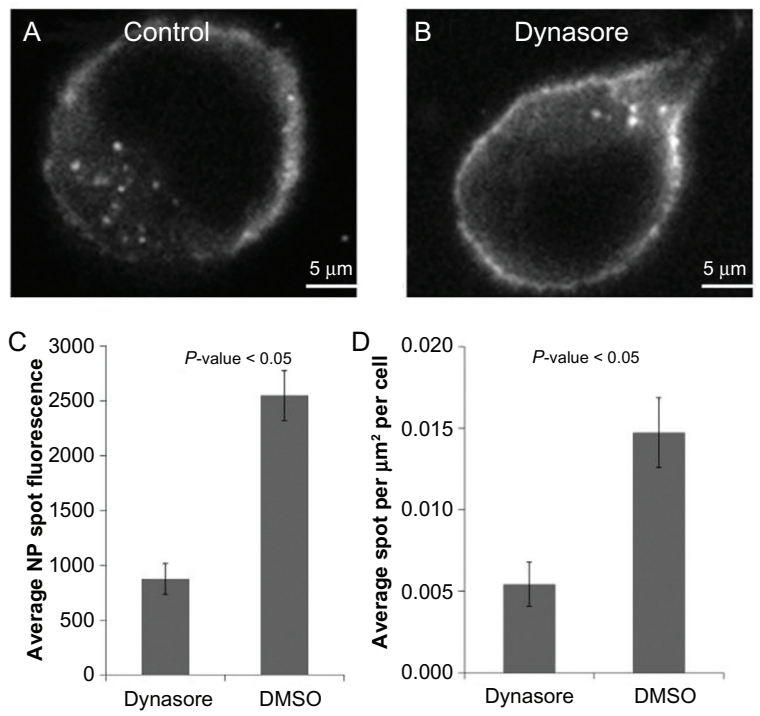

Figure $\mathbf{5}$ In the absence of serum, dynasore treatment results in clear inhibition of nanoparticle entry. Representative images of (A) a HeLa cell incubated with nanoparticles in serum-free media and (B) a HeLa cell incubated with nanoparticles treated with dynasore in serum-free media. (C) Average nanoparticle spot fluorescence and (D) average spot density for HeLa cells incubated with dynasore or dimethyl sulfoxide (control) with nanoparticles in serum-free media.

Abbreviations: DMSO, dimethyl sulfoxide; NP, nanoparticle.
Results from studies in the presence of serum have been omitted as previous studies have shown that serum inhibits dynasore function. ${ }^{21}$

Two main endocytosis pathways regulated by dynamin are clathrin-mediated endocytosis and caveolar endocytosis. ${ }^{19}$ Therefore, one intention of this work was to determine whether each of these might be involved in nanoparticle entry. Importantly, the authors have extensive experience with these inhibitors in endocytosis assays, and have previously shown positive controls for each. ${ }^{13-15,22-24}$ As observed in Figure 6, expression of the inhibitor of clathrin-mediated endocytosis eps15(EH29), ${ }^{25}$ but not dominant negative caveolin $1,{ }^{26}$ significantly reduced nanoparticle uptake. In cells exposed to nanoparticles in serum free media following transfection with either GFP, as control, EH29, to inhibit clathrin-mediated endocytosis, or caveolin1(Y14F), to inhibit caveolar endocytosis, only EH29 inhibited nanoparticle entry. Quantification revealed that both the brightness and density of intracellular nanoparticle accumulations were significantly inhibited by expression of EH29.

Similar observations were obtained in cells expressing these constructs and incubated with nanoparticles in the presence of serum (Figure 7). Interestingly though, although the expression of EH29 significantly reduced the number of nanoparticle accumulations per cell, relative to the control, while decreased quantitatively, the nanoparticle spot fluorescence was not significantly different $(P$ value $=0.058)$. However, expression of caveolin1(Y14F) actually increased the 


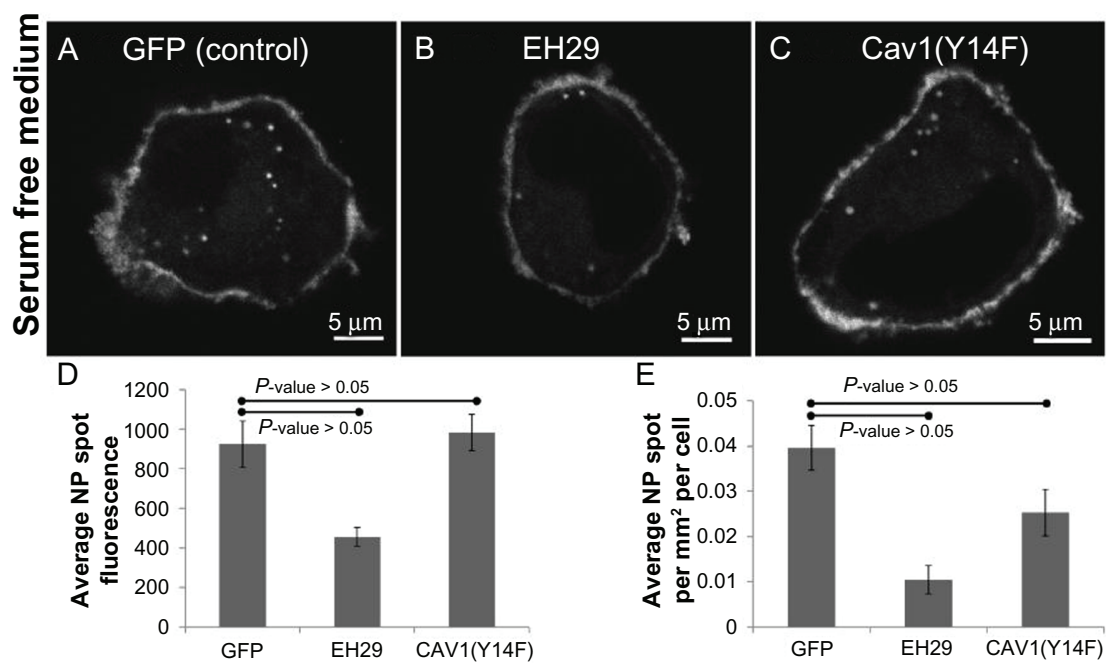

Figure 6 Expression of an inhibitor of clathrin-mediated endocytosis greatly reduces nanoparticle intake whereas inhibiting caveolar endocytosis has no effect compared to a control in serum-free media. Representative images of a HeLa cell expressing (A) green fluorescent protein, (B) EH29, and (C) YI4F incubated with nanoparticles in serum-free media. (D) Average nanoparticle spot fluorescence and (E) average spot density for HeLa cells expressing green fluorescent protein, EH29, and YI4F incubated with nanoparticles in serum-free media.

Abbreviations: GFP, green fluorescent protein; NP, nanoparticle.

fluorescence of the intracellular nanoparticle accumulations relative to the control in this study. Therefore, it is possible that inhibiting caveolar endocytosis increases nanoparticle entry, possibly through a similar mechanism as was recently observed in cells taking up fluorescent dextran. ${ }^{27}$ In this study, expression of this same mutant increased uptake of $70 \mathrm{kDa}$ dextran in ARPE-19 cells. Thus, taken together, these results demonstrate that both in the presence and absence of serum, nanoparticles can enter cells through dynamin-dependent/ clathrin-mediated endocytosis, and that in this system caveolar endocytosis does not seem to be directly involved in nanoparticle entry.

\section{Nanoparticle-mediated cellular permeabilization}

As depicted in Figures 5-7, inhibition of dynamin-dependent/ clathrin-mediated endocytosis significantly, but not completely, inhibited nanoparticle entry into cells. The

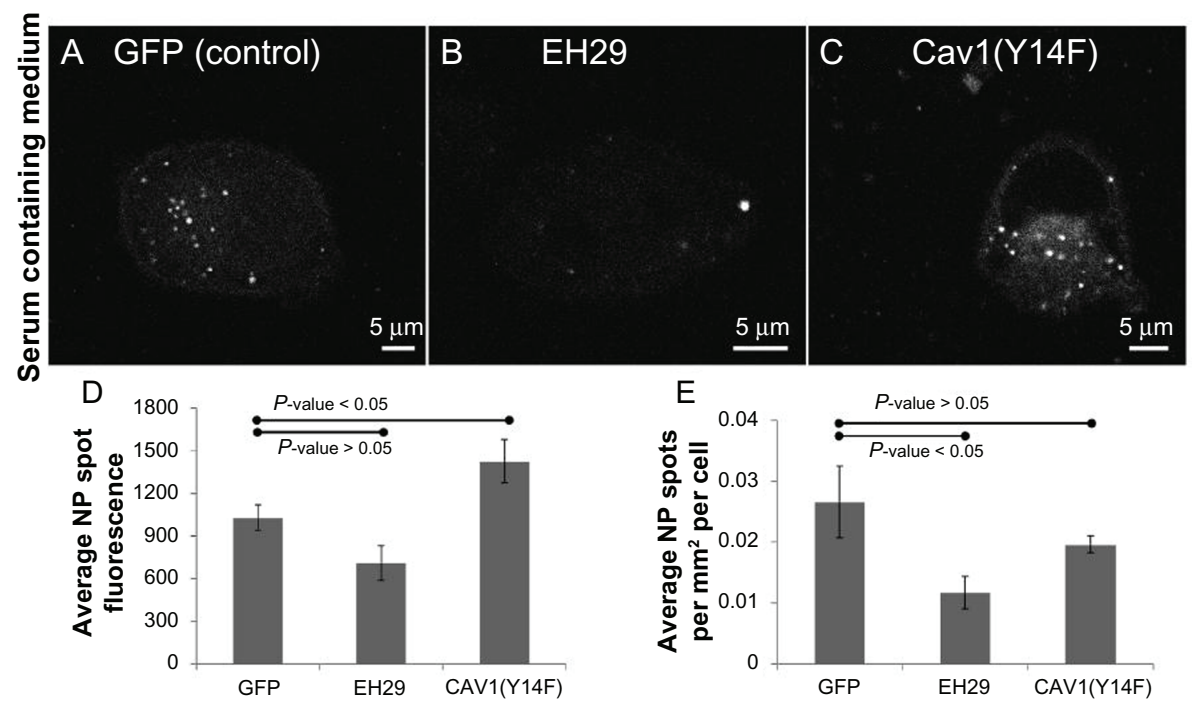

Figure 7 Expression of an inhibitor of clathrin-mediated endocytosis reduces nanoparticle intake, whereas inhibiting caveolar endocytosis does not. Representative images of a HeLa cell expressing (A) green fluorescent protein, (B) EH29, and (C) YI4F incubated with nanoparticles in serum-containing media. (D) Average nanoparticle spot fluorescence and (E) average spot density for HeLa cells expressing green fluorescent protein, EH29, and YI4F incubated with nanoparticles in serum-containing media. Abbreviations: GFP, green fluorescent protein; NP, nanoparticle. 

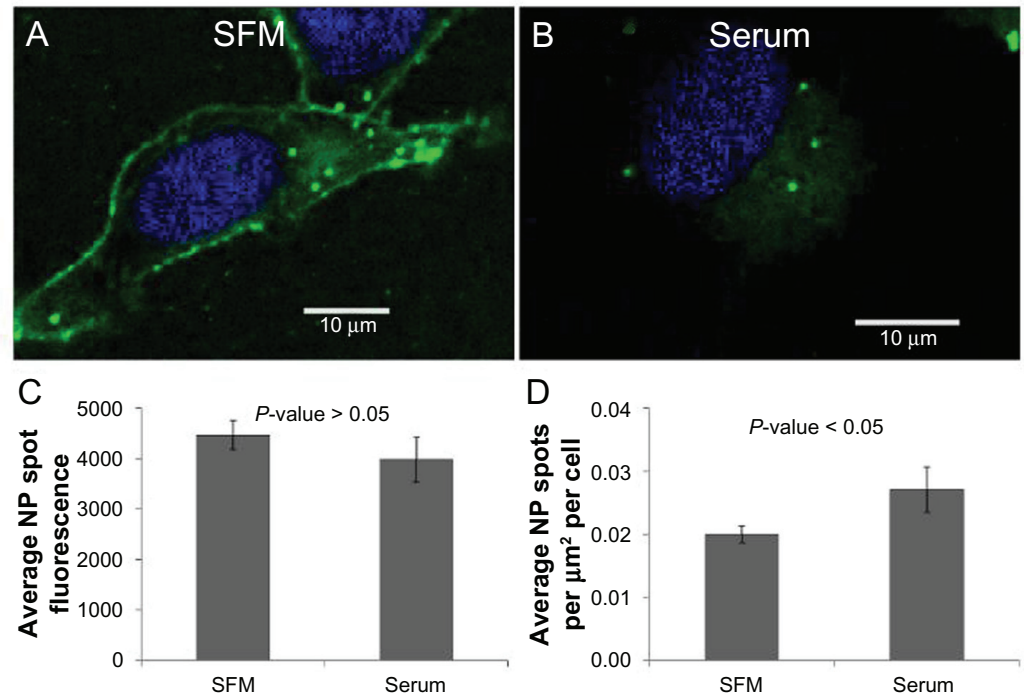

Figure 8 Nanoparticles are able to gain entry to the cytosol via an endocytosis-independent method both in the presence and absence of serum. Representative images of a HeLa cell incubated with nanoparticles in (A) serum-free media and (B) serum-containing media at $4^{\circ} \mathrm{C}$. (C) Average nanoparticle spot fluorescence and (D) average nanoparticle spot density for HeLa cells incubated with nanoparticles in serum-free media at $4^{\circ} \mathrm{C}$.

Abbreviations: NP, nanoparticle; SFM, serum-free media.

proportion of nanoparticle internalization resistant to inhibition of endocytosis could be due to an incomplete inhibition of endocytosis, or it could be that nanoparticles are entering cells through another mechanism. An alternative method for inhibiting endocytosis is to cool cells to $4{ }^{\circ} \mathrm{C},{ }^{28}$ and, as can be seen in Figure 8, at $4^{\circ} \mathrm{C}$ nanoparticles are still able to gain entry into the cytosol. Importantly, these same conditions resulted in a nearly complete inhibition of the internalization

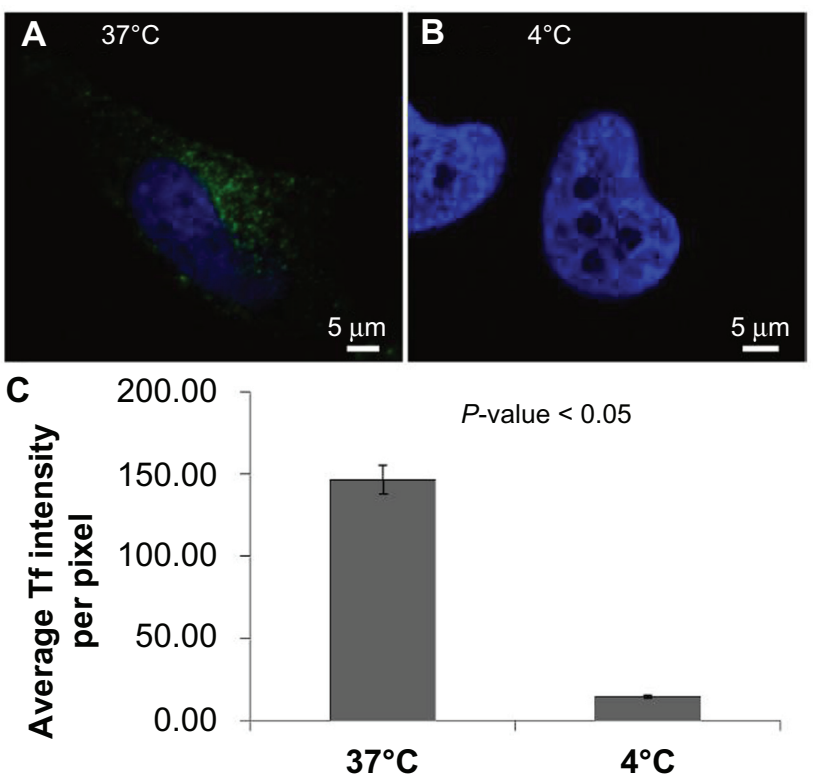

Figure 9 Inhibition of clathrin-mediated endocytosis by incubation of cells in the cold. Representative images of HeLa cells incubated with transferrin at (A) $37^{\circ} \mathrm{C}$ and $(B) 4^{\circ} \mathrm{C}$. Identical imaging conditions and display values demonstrate the nearly complete inhibition of transferrin entry at $4^{\circ} \mathrm{C}$.

Abbreviation: Tf, transferrin. of transferrin, a cargo molecule known to be internalized through clathrin-mediated endocytosis ${ }^{13-15}$ (Figure 9). This might suggest that an endocytosis-independent route for nanoparticle entry exists, particularly when considering that the presence of serum during nanoparticle incubation at $4^{\circ} \mathrm{C}$ does not inhibit entry; in fact, the intracellular density of nanoparticle accumulations was slightly higher compared to cells kept in serum-free media. Given the size and negative charge of the nanoparticles being studied, it is not likely that nanoparticles are able to freely diffuse across the plasma membrane. However, it might be that nanoparticles are entering through discontinuities in the plasma membrane, either preexisting or induced by the nanoparticles through direct permeabilization of the cell surface.

Sytox Green is a commercially available reagent that is membrane impermeant and brightly fluorescent only following binding to nucleic acids. ${ }^{29}$ Therefore, Sytox Green can be used to measure the potential for plasma membrane permeabilization. As depicted in Figure 10, incubation of cells in the presence of nanoparticles at $4^{\circ} \mathrm{C}$ in serum-free and serum-containing media resulted in significantly higher Sytox Green fluorescence than control cells. Strikingly, the extent of Sytox Green fluorescence was roughly equivalent to that observed in cells incubated in the presence of $0.1 \%$ Triton X100, a detergent which is commonly employed to permeabilize the plasma membrane. Thus, these results demonstrate that incubation of cells with $20 \mathrm{~nm}$ carboxylatemodified polystyrene nanoparticles can result in disruption 

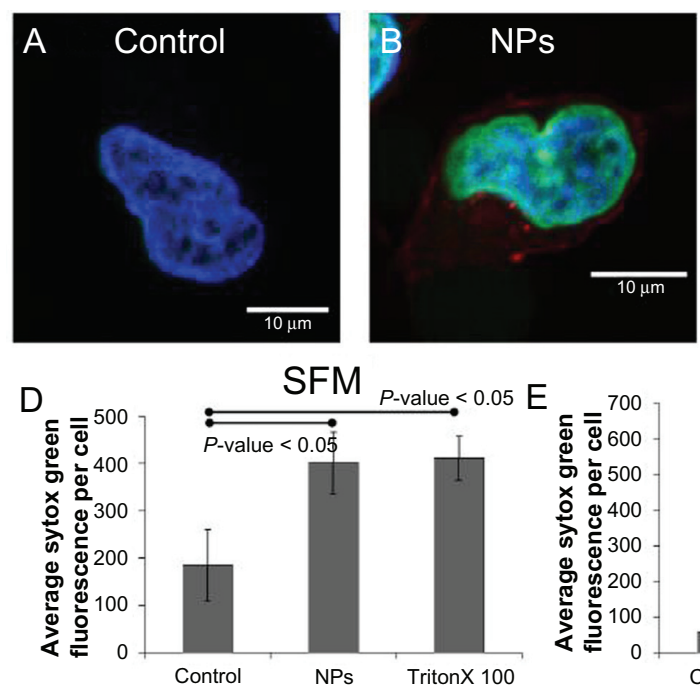

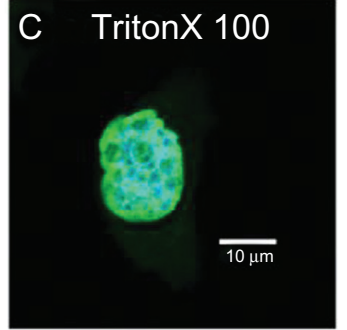

Serum $P$-value $<0.05$

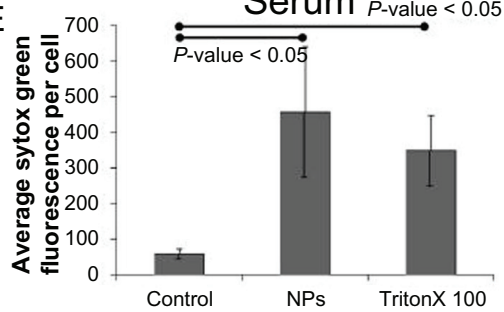

Figure 10 Incubation of HeLa cells in the presence of nanoparticles at $4^{\circ} \mathrm{C}$ in serum-free media resulted in significantly higher Sytox ${ }^{\circledR} \mathrm{Green}$ (Invitrogen Life Technologies, Carlsbad, CA) fluorescence than control cells. Representative images of a HeLa cell incubated with (A) Sytox Green, (B) nanoparticles and Sytox Green, and (C) Triton XI00 and Sytox Green in serum-free media at $4^{\circ} \mathrm{C}$. The average Sytox Green fluorescence per cell for control, nanoparticle-incubated, and detergent-exposed HeLa cells in (D) serum-free media and (E) serum-containing media.

Abbreviations: NPs, nanoparticles; SFM, serum-free media.

of the plasma membrane, and suggest that this might be responsible for a significant proportion of nanoparticle entry in cells.

\section{Discussion}

The increased potential for human exposure to manufactured nanomaterials emphasizes the need for a greater understanding of cellular nanotoxicology. In many cases, particular nanoparticles and target cell types will need to be empirically investigated as varying cell types react in distinct ways to the introduction of nanoparticles, ${ }^{9}$ which themselves display unique behaviors in biological fluid. ${ }^{30}$ However, the use of well-characterized model nanoparticles with known properties is necessary to provide insight into potentially biomedically relevant phenomena. In this study, a detailed analysis of the route of entry for $20 \mathrm{~nm}$ carboxylate-modified polystyrene nanoparticles was performed. The results of the analyses provide exciting new observations which open up new perspectives in the field of cellular nanotoxicology, and suggest several mechanisms relevant to nanoparticle entry that should be considered in future studies.

The first series of experiments consider the potential effects of serum on nanoparticle entry into cultured cells. With a fluorescence plate reader, a striking reduction in cellular-associated nanoparticle signal was observed when cells were incubated with nanoparticles in serum-containing, versus serum-free, conditions. Furthermore, this was observed with cells from different organisms, and in both epithelial and transformed cells. A more detailed analysis through confocal microscopy revealed that the presence of serum both reduced nanoparticle signal at the plasma membrane and intracellular accumulation. Therefore, serum appears to be able to reduce the ability of nanoparticles to bind to and enter cells, observations previously alluded to with the use of larger (eg, $\sim 64 \mathrm{~nm}$ ) positively-charged nanoparticles. ${ }^{10}$

Next, several potential mechanisms for nanoparticle internalization into cells were evaluated. In both serum-free and serum-containing studies, it was demonstrated that these nanoparticles can enter cells through dynamin-dependent/ clathrin-mediated endocytosis, and not caveolar endocytosis. However, the presence of endocytosis-independent nanoparticle uptake was also able to be demonstrated. The analyses demonstrate that $20 \mathrm{~nm}$ carboxylate-modified polystyrene nanoparticles have the capacity to induce plasma membrane permeabilization. Therefore, this may reflect an alternative means by which nanoparticles are able to gain entry to the intracellular space, and potentially cause cellular damage.

In the current study, it was demonstrated that nanoparticle interaction with, and subsequent entry into cells, is significantly inhibited by the presence of serum. It has been noted that a highly fluidic protein corona with a rapid exchange rate is less likely to adsorb to the plasma membrane, ${ }^{16}$ possibly due to the resulting reduced zeta potential. Furthermore, carboxy functionalization of nanoparticles has been proposed to be more amiable to nanoparticle-protein interactions than amino modifications. ${ }^{11}$ Finally, the relationship between decreased membrane adsorption and reduced uptake of nanoparticles has been previously suggested. ${ }^{10,17}$ 

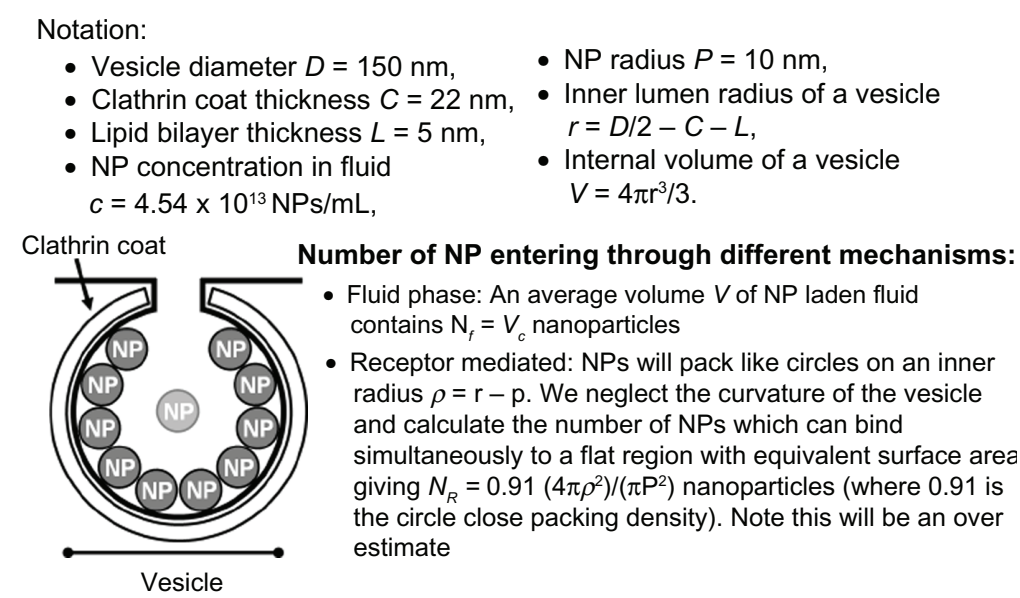

Average no of NPs in lumen: $\mathrm{N}_{f}=2.1 \times 10^{-3}$ Max no of NPs on inner surface: $\mathrm{N}_{R}=52$

Figure I I A series of calculations to estimate the number of nanoparticles that could enter a vesicle under the incubation conditions described in materials and methods. It is estimated that nanoparticles are nearly 25,000 times more likely to enter cells when they are bound to the membrane than in the fluid phase.

Abbreviation: NP, nanoparticle.

Taken together, this suggests that the addition of serum generates a highly fluidic protein corona with a rapid exchange rate which prevents absorption to the plasma membrane and subsequent internalization.

In order to quantitatively assess the relative potential for nanoparticle entry through the fluid phase, dispersed in the lumen of a vesicle, as opposed to through receptor-mediated means, adhered to the inner membrane of the vesicle, a series of calculations was performed to estimate the number of nanoparticles that could enter a vesicle under the incubation conditions described above (Figure 11). This analysis assumed a total vesicle diameter of $150 \mathrm{~nm},{ }^{31}$ and that the entire cell surface has the capacity to be coated in nanoparticles. Even though the latter might be an overestimation, the current analyses suggest that under these conditions nanoparticles are nearly 25,000 times more likely to enter cells bound to the inner membrane of a vesicle than through the fluid phase. Thus, when taken together, the data suggest that cellular entry of $20 \mathrm{~nm}$ carboxylate-modified polystyrene nanoparticles is reduced in the presence of serum primarily through inhibition of cell surface binding, that clathrin-mediated endocytosis represents a significant route for nanoparticle uptake, and that these nanoparticles potentially have the capacity to enter the cell directly through permeabilization of the plasma membrane.

\section{Conclusion}

In summary, the current report analyzes the cellular internalization of model nanoparticles, focusing on factors which might affect the association of nanoparticles with cells and possible routes of entry into cells. It has been established that the presence of serum in media greatly decreases cellular nanoparticle fluorescence intensity. This was shown to be neither cell type nor species dependent. Furthermore, it was shown that nanoparticle entry into cells was greatly reduced when inhibiting the dynamin-dependent/clathrin-mediated endocytosis pathway. Finally, using a membrane impermeant nucleic acid stain, it was shown that the presence of nanoparticles permeabilizes the plasma membrane to a similar extent as a detergent, Triton X100. This would suggest that permeabilization of the plasma membrane may be responsible for a proportion of nanoparticle entry into cells.

\section{Executive summary}

- Cells were incubated with $20 \mathrm{~nm}$ carboxylate-modified polystyrene nanoparticles in the presence of serumcontaining and serum-free media.

- Serum-containing media was found to decrease intracellular nanoparticle accumulation, primarily through inhibition of cell surface binding.

- Clathrin-mediated endocytosis represents a significant route for nanoparticle uptake.

- These nanoparticles potentially have the capacity to enter the cell directly through permeabilization of the cell membrane.

\section{Acknowledgments}

The authors would like to thank Professor Mark McNiven (The Mayo Clinic, Rochester, MN) for caveolin1(Y14F) and Dr Alexandre Benmerah (Institut Cochin, Paris, France) for eps15(EH29). JM is funded through Natural Environment 
Research Council/Medical Research Council Project grant NE/I008314/1; PJS is funded by the Engineering and Physical Sciences Research Council through the Physical Sciences of Imaging for the Biomedical Sciences Doctoral Training Center. Finally, the authors would like to thank the following people for technical assistance and contributions to assay development: Adam Williams, Bhupesh Panchal, Georgina Harris, and Jade Ogle, as well as members of the 2011 United Kingdom Mathematics-in-Medicine Study Group for assistance with computational analyses. . The authors would also like to thank the Facility for Environmental Nanoscience Analysis and Characterisation (FENAC).

\section{Disclosure}

The authors report no conflicts of interest in this work.

\section{References}

1. Auffan M, Rose J, Bottero JY, Lowry GV, Jolivet JP, Wiesner MR Towards a definition of inorganic nanoparticles from an environmental, health and safety perspective. Nat Nanotechnol. 2009;4(10):634-641.

2. Maynard AD, Aitken RJ, Butz T, et al. Safe handling of nanotechnology. Nature. 2006;444(7117):267-269.

3. Madl AK, Pinkerton KE. Health effects of inhaled engineered and incidental nanoparticles. Crit Rev Toxicol. 2009;39(8):629-658.

4. Nel AE, Madler L, Velegol D, et al. Understanding biophysicochemical interactions at the nano-bio interface. Nat Mater. 2009;8(7):543-557.

5. Dausend J, Musyanovych A, Dass M, et al. Uptake mechanism of oppositely charged fluorescent nanoparticles in HeLa cells. Macromol Biosci. 2008;8(12):1135-1143.

6. Xia T, Kovochich M, Liong M, Zink JI, Nel AE. Cationic polystyrene nanosphere toxicity depends on cell-specific endocytic and mitochondrial injury pathways. ACS Nano. 2008;2(1):85-96.

7. Rejman J, Oberle V, Zuhorn IS, Hoekstra D. Size-dependent internalization of particles via the pathways of clathrin- and caveolae-mediated endocytosis. Biochem J. 2004;377(Pt 1):159-169.

8. Johnston HJ, Semmler-Behnke M, Brown DM, Kreyling W, Tran L, Stone V. Evaluating the uptake and intracellular fate of polystyrene nanoparticles by primary and hepatocyte cell lines in vitro. Toxicol Appl Pharmacol. 2010;242(1):66-78.

9. Dos Santos T, Varela J, Lynch I, Salvati A, Dawson KA. Effects of transport inhibitors on the cellular uptake of carboxylated polystyrene nanoparticles in different cell lines. PLoS One. 2011;6(9):e24438.

10. Merhi M, Dombu CY, Brient A, et al. Study of serum interaction with a cationic nanoparticle: implications for in vitro endocytosis, cytotoxicity and genotoxicity. Int J Pharm. 2012;423(1):37-44.

11. Gessner A, Lieske A, Paulke BR, Muller RH. Functional groups on polystyrene model nanoparticles: influence on protein adsorption. J Biomed Mater Res A. 2003;65(3):319-326.

12. Baier G, Costa C, Zeller A, et al. BSA adsorption on differently charged polystyrene nanoparticles using isothermal titration calorimetry and the influence on cellular uptake. Macromol Biosci. 2011;11(5):628-638.

International Journal of Nanomedicine

\section{Publish your work in this journal}

The International Journal of Nanomedicine is an international, peerreviewed journal focusing on the application of nanotechnology in diagnostics, therapeutics, and drug delivery systems throughout the biomedical field. This journal is indexed on PubMed Central, MedLine, CAS, SciSearch $\AA$, Current Contents ${ }^{\circledR} /$ Clinical Medicine,
13. Rappoport JZ, Taha BW, Lemeer S, Benmerah A, Simon SM. The AP-2 complex is excluded from the dynamic population of plasma membraneassociated clathrin. J Biol Chem. 2003;278(48):47357-47360.

14. Rappoport JZ, Simon SM. A functional GFP fusion for imaging clathrinmediated endocytosis. Traffic. 2008;9(8):1250-1255.

15. Rappoport JZ, Simon SM. Endocytic trafficking of activated EGFR is AP-2 dependent and occurs through preformed clathrin spots. J Cell Sci. 2009;122(Pt 9):1301-1305.

16. Lynch I, Salvati A, Dawson KA. Protein-nanoparticle interactions: what does the cell see? Nat Nanotechnol. 2009;4(9):546-547.

17. Wilhelm C, Gazeau F, Roger J, et al. Binding of biological effectors on magnetic nanoparticles measured by a magnetically induced transient birefringence experiment. Phys Rev E Stat Nonlin Soft Matter Phys. 2002;65(3 Pt 1):031404.

18. Lesniak A, Campbell A, Monopoli MP, Lynch I, Salvati A, Dawson KA. Serum heat inactivation affects protein corona composition and nanoparticle uptake. Biomaterials. 2010;31(36):9511-9518.

19. Doherty GJ, McMahon HT. Mechanisms of endocytosis. Annu Rev Biochem. 2009;78:857-902.

20. Macia E, Ehrlich M, Massol R, Boucrot E, Brunner C, Kirchhausen T. Dynasore, a cell-permeable inhibitor of dynamin. Dev Cell. 2006;10(6): 839-850.

21. Kirchhausen T, Macia E, Pelish HE. Use of dynasore, the small molecule inhibitor of dynamin, in the regulation of endocytosis. Methods Enzymol. 2008;438:77-93.

22. Farquhar MJ, Hu K, Harris HJ, et al. Hepatitis C virus induces CD81 and claudin-1 endocytosis. J Virol. February 8, 2012. [Epub ahead of print.]

23. Fletcher SJ, Poulter NS, Haining EJ, Rappoport JZ. Clathrin-mediated endocytosis regulates occludin, and not focal adhesion, distribution during epithelial wound healing. Biol Cell. December 19, 2011. [Epub ahead of print.]

24. Rappoport JZ, Kemal S, Benmerah A, Simon SM. Dynamics of clathrin and adaptor proteins during endocytosis. Am J Physiol Cell Physiol. 2006;291(5):C1072-C1081.

25. Benmerah A, Bayrou M, Cerf-Bensussan N, Dautry-Varsat A. Inhibition of clathrin-coated pit assembly by an Eps15 mutant. J Cell Sci. 1999;112(Pt 9):1303-1311.

26. Orlichenko L, Huang B, Krueger E, McNiven MA. Epithelial growth factorinduced phosphorylation of caveolin 1 at tyrosine 14 stimulates caveolae formation in epithelial cells. J Biol Chem. 2006;281(8): 4570-4579.

27. Vercauteren D, Piest M, van der Aa LJ, et al. Flotillin-dependent endocytosis and a phagocytosis-like mechanism for cellular internalization of disulfide-based poly(amido amine)/DNA polyplexes. Biomaterials . 2011;32(11):3072-3084.

28. Goldenthal KL, Pastan I, Willingham MC. Initial steps in receptormediated endocytosis. The influence of temperature on the shape and distribution of plasma membrane clathrin-coated pits in cultured mammalian cells. Exp Cell Res. 1984;152(2):558-564.

29. Roth BL, Poot M, Yue ST, Millard PJ. Bacterial viability and antibiotic susceptibility testing with SYTOX green nucleic acid stain. Appl Environ Microbiol. 1997;63(6):2421-2431.

30. Safi M, Courtois J, Seigneuret M, Conjeaud H, Berret JF. The effects of aggregation and protein corona on the cellular internalization of iron oxide nanoparticles. Biomaterials. 2011;32(35):9353-9363.

31. McMahon HT, Boucrot E. Molecular mechanism and physiological functions of clathrin-mediated endocytosis. Nat Rev Mol Cell Biol. 2011;12(8):517-533.

\section{Dovepress}

Journal Citation Reports/Science Edition, EMBase, Scopus and the Elsevier Bibliographic databases. The manuscript management system is completely online and includes a very quick and fair peer-review system, which is all easy to use. Visit http://www.dovepress.com/ testimonials.php to read real quotes from published authors. 